PROCEEDINGS OF THE

AMERICAN MATHEMATICAL SOCIETY

Volume 132, Number 4, Pages 1013-1019

S 0002-9939(03)07301-5

Article electronically published on November 10, 2003

\title{
ASYMPTOTIC ESTIMATES FOR A TWO-DIMENSIONAL PROBLEM WITH POLYNOMIAL NONLINEARITY
}

\author{
ADIMURTHI AND MASSIMO GROSSI \\ (Communicated by David S. Tartakoff)
}

\begin{abstract}
In this paper we give asymptotic estimates of the least energy solution $u_{p}$ of the functional

$$
J(u)=\int_{\Omega}|\nabla u|^{2} \quad \text { constrained on the manifold } \int_{\Omega}|u|^{p+1}=1
$$

as $p$ goes to infinity. Here $\Omega$ is a smooth bounded domain of $\mathbb{R}^{2}$. Among other results we give a positive answer to a question raised by Chen, $\mathrm{Ni}$, and Zhou (2000) by showing that $\lim _{p \rightarrow \infty}\left\|u_{p}\right\|_{\infty}=\sqrt{e}$.
\end{abstract}

\section{INTRODUCTION}

In this paper we consider the following elliptic minimization problem. Let us define a $C^{2}$-functional on $H_{0}^{1}(\Omega)$ :

$$
J(u)=\int_{\Omega}|\nabla u|^{2} \quad \text { constrained on the manifold } \int_{\Omega}|u|^{p+1}=1
$$

where $\Omega$ is a smooth bounded domain in $\mathbb{R}^{2}$ and $p$ is a real number greater than 1 . Then we define

$$
S_{p}=\inf _{u \in H_{0}^{1}(\Omega)} J(u)
$$

By standard results it is easy to see that $S_{p}$ is achieved at a function $u_{p} \in H_{0}^{1}(\Omega)$ that satisfies

$$
\left\{\begin{array}{cc}
-\Delta u_{p}=S_{p} u_{p}^{p} & \text { in } \Omega \\
u_{p}>0 & \text { in } \Omega \\
u_{p}=0 & \text { on } \partial \Omega .
\end{array}\right.
$$

By Lemma 2.1 we get $S_{p}=O\left(\frac{1}{p}\right)$ for $p$ large. Setting $v_{p}=S_{p}^{\frac{1}{p-1}} u_{p}$ we are in the framework of [8], [9] and [6] where some asymptotic results about this problem were obtained.

Received by the editors September 7, 2002.

2000 Mathematics Subject Classification. Primary 35J20, 35B40.

Supported by M.U.R.S.T., project "Variational methods and nonlinear differential equations".

(C)2003 American Mathematical Society 
In particular, it was proved in [8] and [9] that the minimizer $u_{p}$ looks like a sharp "spike". More precisely it was shown that, for a suitable sequence $p_{n} \rightarrow \infty$,

$$
\left\|u_{p_{n}}\right\|_{\infty} \leq C
$$

and in the sense of distribution,

$$
\frac{u_{p_{n}}^{p_{n}}}{\left\|u_{p_{n}}\right\|_{p_{n}}^{p_{n}}} \rightarrow \delta_{x_{0}}
$$

Moreover, the point $x_{p_{n}}$ where the minimizer $u_{p_{n}}$ achieves its maximum converges to a critical point of the Robin function.

In this paper we obtain estimates of a different nature, greatly improving some partial results obtained in [5], where uniqueness and qualitative properties of the least energy solution were proved.

Here we use the two-dimensional blow-up technique introduced in [1. The blowup function is obtained by linearizing the nonlinear term plog $u_{p}$ around the point of maximum of $u_{p}$. More precisely let us define the function $z_{p}(x): \Omega_{p}=\frac{\Omega-x_{p}}{\varepsilon_{p}} \mapsto \mathbb{R}$,

$$
z_{p}(x)=\frac{p}{u_{p}\left(x_{p}\right)}\left(u_{p}\left(\varepsilon_{p} x+x_{p}\right)-u_{p}\left(x_{p}\right)\right)
$$

where $x_{p}$ is the point where $u_{p}$ achieves its maximum and $\varepsilon_{p}^{2}=\frac{1}{p S_{p} u_{p}\left(x_{p}\right)^{p-1}}$. Then we obtain the following.

Theorem 1.1. For any sequence $z_{p_{n}}$ with $p_{n} \rightarrow \infty$, there exists a subsequence of $z_{p_{n}}$, still denoted by $z_{p_{n}}$, such that $z_{p_{n}} \rightarrow z$ in $C_{\text {loc }}^{2}\left(\mathbb{R}^{2}\right)$, where $z(x)=\log \frac{1}{\left(1+\frac{|x|^{2}}{8}\right)^{2}}$.

The main result of the paper is a consequence of the above theorem.

Theorem 1.2. Let $u_{p}$ be a solution to (1.3). Then

$$
\lim _{p \rightarrow \infty}\left\|u_{p}\right\|_{\infty}=\sqrt{e}
$$

Note that the estimate $\limsup _{p \rightarrow \infty}\left\|u_{p}\right\|_{\infty} \leq \sqrt{e}$ was proved in [9]. Here we give a positive answer to a question raised in [4, where some numerical computations suggested the validity of (1.7).

\section{Proof of Theorem 1.1}

In this section we recall some results about the solution $u_{p}$, and then we give the proof of Theorem 1.1

We start by recalling the following estimates on $S_{p}$, due to Ren and Wei ([8]).

Lemma 2.1. Let $S_{p}$ be defined as in (1.2). Then

$$
\lim _{p \rightarrow \infty} p S_{p}=8 \pi e \text {. }
$$

Proof. Setting $v_{p}=\left(S_{p}\right)^{\frac{1}{p-1}} u_{p}$ we have that $v_{p}$ also achieves $S_{p}$ and satisfies

$$
\left\{\begin{array}{cc}
-\Delta v_{p}=v_{p}^{p} & \text { in } \Omega, \\
v_{p}>0 & \text { in } \Omega, \\
v_{p}=0 & \text { on } \partial \Omega .
\end{array}\right.
$$

From Corollary 2.3 of [8] we get $\lim _{p \rightarrow \infty} p \int_{\Omega} v_{p}^{p+1}=8 \pi e$. Hence, recalling that $\int_{\Omega} u_{p}^{p+1}=1$ we derive that $\lim _{p \rightarrow \infty} p\left(S_{p}\right)^{\frac{p+1}{p-1}}=8 \pi e$, which implies 2.1). 
Let us denote by $x_{p}$ the point where $u_{p}\left(x_{p}\right)=\left\|u_{p}\right\|_{\infty}$. By Lemma 4.1 of [8] we know that $x_{p}$ is far away from the boundary of $\Omega$. The next lemma provides additional information on the rate of $u_{p}\left(x_{p}\right)$.

Lemma 2.2. We have that

$$
\lim _{p \rightarrow \infty} u_{p}\left(x_{p}\right)^{p-1}=+\infty .
$$

Proof. Let us denote by $\lambda_{1}(\Omega)$ the first eigenvalue of $-\Delta$ with zero Dirichlet boundary condition. Then we have

$$
1=\int_{\Omega}\left|u_{p}\right|^{p+1} \leq u_{p}\left(x_{p}\right)^{p-1} \int_{\Omega}\left|u_{p}\right|^{2} \leq u_{p}\left(x_{p}\right)^{p-1} \lambda_{1}(\Omega)^{-1} \int_{\Omega}\left|\nabla u_{p}\right|^{2} .
$$

Recalling that $\int_{\Omega}\left|\nabla u_{p}\right|^{2}=S_{p}$ and Lemma 2.1, we deduce that $\int_{\Omega}\left|\nabla u_{p}\right|^{2} \rightarrow 0$ as $p$ goes to infinity. By (2.4), we obtain the claim.

Proof of Theorem [1.1. For any sequence $p_{n} \rightarrow+\infty$, let us set $z_{n}: \Omega_{n}=\frac{\Omega-x_{p_{n}}}{\varepsilon_{n}} \mapsto$ $\mathbb{R}$

$$
z_{n}(x)=\frac{p_{n}}{u_{p_{n}}\left(x_{p_{n}}\right)}\left(u_{p_{n}}\left(\varepsilon_{n} x+x_{p_{n}}\right)-u_{p_{n}}\left(x_{p_{n}}\right)\right)
$$

where $\varepsilon_{n}^{2}=\frac{1}{p_{n} S_{p_{n}} u_{p_{n}}\left(x_{p_{n}}\right)^{p_{n}-1}}$. From Lemma 2.1 and Lemma 2.2, we get $\varepsilon_{n} \rightarrow 0$ as $n \rightarrow \infty$ and " $\Omega_{n} \rightarrow \mathbb{R}^{2}$ " as $n \rightarrow \infty$. Now let us write down the equation satisfied by $z_{n}$,

$$
\left\{\begin{array}{cc}
-\Delta z_{n}=\left(1+\frac{z_{n}}{p_{n}}\right)^{p_{n}} & \text { in } \Omega_{n}, \\
0<1+\frac{z_{n}}{p_{n}} \leq 1 & \text { in } \Omega_{n}, \\
z_{n}=-p_{n} & \text { on } \partial \Omega_{n} .
\end{array}\right.
$$

We want to pass to the limit in (2.6). To do this we use some ideas in 2]. Let $B(0, R)$ be the ball centered at the origin with radius $R$, and let $w_{n}$ be the solution of

$$
\left\{\begin{array}{cc}
-\Delta w_{n}=\left(1+\frac{z_{n}}{p_{n}}\right)^{p_{n}} & \text { in } B(0, R), \\
w_{n}=0 & \text { on } \partial B(0, R) .
\end{array}\right.
$$

By the maximum principle and the standard regularity theory, we have that $0 \leq w_{n} \leq C$ with $C$ independent of $n$. For $x \in B(0, R)$ set $\psi_{n}(x)=z_{n}(x)-w_{n}(x)$. Hence $\psi_{n}$ is a sequence of harmonic functions which are uniformly bounded above. Hence by Harnack's inequality [7] we have the alternative: either i) a subsequence of $\psi_{n}$ is bounded in $L_{l o c}^{\infty}(B(0, R))$,

or

ii) $\psi_{n}$ converges uniformly to $-\infty$ on compact subsets of $(B(0, R)$.

Since $\psi_{n}(0)=z_{n}(0)-w_{n}(0)=-w_{n}(0) \geq-C$, case ii) cannot occur. Hence, up to a subsequence, which we denote again by $\psi_{n}$, we have that $\psi_{n}$ is bounded in $L^{\infty}(B(0, R))$ for any $R>0$ and the same holds for $z_{n}$. From (2.6), and the standard regularity theory, we derive that $z_{n}$ is bounded in $C_{l o c}^{2}\left(\mathbb{R}^{2}\right)$, and then it converges to a function $z \in C^{2}\left(\mathbb{R}^{2}\right)$. Passing to the limit in (2.6), we get that $z$ satisfies

$$
-\Delta z=e^{z} \quad \text { in } \mathbb{R}^{2} .
$$


Let us prove that $\int_{\mathbb{R}^{2}} e^{z}<+\infty$. To do this we observe that, since $z_{n} \rightarrow z$ in $C_{l o c}^{2}\left(\mathbb{R}^{2}\right)$, then

$$
p_{n}\left(\log \left(1+\frac{z_{n}}{p_{n}}\right)-\frac{z_{n}}{p_{n}}\right) \rightarrow 0 \quad \text { pointwise in } \mathbb{R}^{2} .
$$

Hence

$$
z_{n}+p_{n}\left(\log \left(1+\frac{z_{n}}{p_{n}}\right)-\frac{z_{n}}{p_{n}}\right) \rightarrow z \quad \text { pointwise in } \mathbb{R}^{2} .
$$

By Fatou's Lemma, we deduce

$$
\begin{aligned}
\int_{\mathbb{R}^{2}} e^{z} & \leq \lim _{n \rightarrow \infty} \int_{\Omega_{n}} e^{z_{n}+p_{n}\left(\log \left(1+\frac{z_{n}}{p_{n}}\right)-\frac{z_{n}}{p_{n}}\right)}=\lim _{n \rightarrow \infty} \int_{\Omega_{n}}\left(1+\frac{z_{n}}{p_{n}}\right)^{p_{n}} \\
& =\lim _{n \rightarrow \infty} \frac{1}{\varepsilon_{n}^{2} u_{p_{n}}^{p_{n}}\left(x_{p_{n}}\right)} \int_{\Omega} u_{p_{n}}^{p_{n}} \leq \lim _{n \rightarrow \infty} \frac{p_{n} S_{p_{n}}}{u_{p_{n}}\left(x_{p_{n}}\right)}|\Omega|^{\frac{1}{p_{n}+1}} \leq C
\end{aligned}
$$

since $u_{p_{n}}\left(x_{p_{n}}\right) \geq C$ in $\Omega$ for $n$ large (see [8], p. 755).

By a result of Chen and $\operatorname{Li}([3])$, the solutions of (2.8) satisfying $\int_{\mathbb{R}^{2}} e^{z}<+\infty$ are given by

$$
z(x)=\log \frac{\mu}{\left(1+\frac{\mu}{8}\left|x-x_{0}\right|^{2}\right)^{2}} \quad \text { for } \mu>0 \text { and } x_{0} \in \mathbb{R}^{2} .
$$

Since $z(x) \leq z(0)=0$ for any $x \in \mathbb{R}^{2}$, we derive that $\mu=1$ and $x_{0}=0$ in (2.12), and this gives the claim of Theorem 1.1

\section{Proof of Theorem 1.2}

The next estimate plays a role in the proof of Theorem 1.2. This estimate was proved in [9] but we stress that it follows easily by Theorem 1.1 .

Lemma 3.1. We have that

$$
\limsup _{n \rightarrow \infty}\left\|u_{p_{n}}\right\|_{\infty} \leq \sqrt{e} .
$$

Proof. It follows directly by Theorem 1.1.

Setting $u_{p_{n}}=u_{n}$ and $L=\limsup _{n \rightarrow \infty}\left\|u_{n}\right\|_{\infty}$, by using Fatou's Lemma, we obtain

$$
\begin{aligned}
1 & =\int_{\Omega} u_{n}^{p+1}=u_{n}\left(x_{n}\right)^{p_{n}+1} \varepsilon_{n}^{2} \int_{\Omega_{n}}\left(1+\frac{z_{n}}{p_{n}}\right)^{p_{n}+1} \\
& =\frac{u_{n}\left(x_{n}\right)^{2}}{p_{n} S_{p_{n}}} \int_{\Omega_{n}}\left(1+\frac{z_{n}}{p_{n}}\right)^{p_{n}+1} \geq \frac{L^{2}}{8 \pi e} \int_{\mathbb{R}^{2}} e^{z} .
\end{aligned}
$$

Recalling that $\int_{\mathbb{R}^{2}} e^{z}=8 \pi$, we deduce the claim.

Let us consider the linearized operator associated to (1.3), i.e., $L_{p}: H_{0}^{1}(\Omega) \rightarrow$ $H^{-1}(\Omega)$,

$$
L_{p}=-\Delta-p S_{p} u_{p}^{p-1}(x) I, \quad x \in \Omega,
$$

and let us denote by $\lambda_{1}\left(L_{p}\right), \lambda_{2}\left(L_{p}\right)$ the first and the second eigenvalue of $L_{p}$. Now let us recall a property of $\lambda_{2}\left(L_{p}\right)$.

Lemma 3.2. We have that

$$
\lambda_{2}\left(L_{p}\right) \geq 0 .
$$


Proof. The proof is standard since $u_{p}$ is a minimizer of $J$ on the manifold $\int_{\Omega}|u|^{p+1}=$ 1.

We consider, for $D \subset \Omega_{p}, L_{p, D}: H_{0}^{1}(D) \rightarrow H^{-1}(D)$,

$$
L_{p, D}=-\Delta-\frac{u_{p}^{p-1}\left(\varepsilon_{p} x+x_{p}\right)}{u_{p}^{p-1}\left(x_{p}\right)} I, \quad x \in D,
$$

and let us denote by $\lambda_{1}\left(L_{p, D}\right), \lambda_{2}\left(L_{p, D}\right)$ the first and the second eigenvalue of $L_{p, D}$.

Lemma 3.3. We have that

$$
\lambda_{2}\left(L_{p, \Omega_{p}}\right) \geq 0 \text {. }
$$

Proof. Using the scaling $x \rightarrow \varepsilon_{p} x+x_{p}$ we get $\lambda_{2}\left(L_{p, \Omega_{p}}\right)=\varepsilon_{p}^{2} \lambda_{2}\left(L_{p}\right)$ and (3.6) follows by Lemma 3.2 .

Lemma 3.4. Let us denote by $B_{1}=B(0,1)$. Let $p_{n} \rightarrow \infty$ such that $z_{p_{n}} \rightarrow z$ in $C_{\text {loc }}^{1}\left(\mathbb{R}^{2}\right)$. Then for large $p_{n}$, we have

$$
\lambda_{1}\left(L_{p_{n}, B_{1}}\right)<0
$$

Proof.

$$
w_{p}=x \cdot \nabla z_{p}+\frac{2}{p-1} z_{p}+\frac{2 p}{p-1} .
$$

By direct computation we get that $w_{p}$ satisfies

$$
-\Delta w_{p}=\frac{u_{p}^{p-1}\left(\varepsilon_{p} x+x_{p}\right)}{u_{p}^{p-1}\left(x_{p}\right)} w_{p} .
$$

Moreover, $w_{p_{n}}(0) \rightarrow 2$ and for $|x|=1, w_{p_{n}}(x) \rightarrow-\frac{4}{9}$ as $p_{n} \rightarrow \infty$.

Hence, if we denote by $A_{p}=\left\{x \in B_{1}: w_{p}>0\right\}$ and

$$
\tilde{w}_{p}= \begin{cases}w_{p} & \text { if } x \in A_{p}, \\ 0 & \text { if } x \in B_{1} \backslash \bar{A}_{p}\end{cases}
$$

we derive that for $p_{n}$ large, $\tilde{w}_{p_{n}} \in H_{0}^{1}\left(B_{1}\right)$. From w.9 wet

$$
\int_{B_{1}}\left|\nabla \tilde{w}_{p_{n}}\right|^{2}-\int_{B_{1}} \frac{u_{p_{n}}^{p_{n}-1}\left(\varepsilon_{p_{n}} x+x_{p_{n}}\right)}{u_{p_{n}}^{p_{n}-1}\left(x_{p_{n}}\right)} \tilde{w}_{p_{n}}^{2}=0,
$$

and this implies that $\lambda_{1}\left(L_{p_{n}, B_{1}}\right)<0$.

Lemma 3.5. Let $p_{n}$ be a sequence as in Lemma 3.4. Then for $p_{n}$ large we have

$$
\lambda_{1}\left(L_{p_{n}, \Omega_{p_{n}} \backslash B_{1}}\right)>0 .
$$

Proof. By contradiction let us suppose that $\lambda_{1}\left(L_{p_{n}, \Omega_{p_{n}} \backslash B_{1}}\right) \leq 0$. Then from Lemma 3.4 , for large $p_{n}, \lambda_{1}\left(L_{p_{n}, B_{1}}\right)<0$ and hence $\lambda_{2}\left(L_{p_{n}, \Omega_{p_{n}}}\right)<0$. This gives a contradiction with Lemma 3.3

Remark 3.6. Lemma 3.4 implies that the operator $L_{p_{n}}, \Omega_{p_{n} \backslash B_{1}}$ satisfies the maximum principle in $\Omega_{p_{n}} \backslash B_{1}$. 
Proof of Theorem 1.2, By Lemma 3.1 we know that (up to a subsequence) $\lim _{n \rightarrow \infty}\left\|u_{p_{n}}\right\|_{\infty} \leq \sqrt{e}$. By contradiction let us suppose that there exists a subsequence of $u_{p_{n}}$ (still denoted by $u_{p_{n}}$ ) such that

$$
\lim _{n \rightarrow \infty}\left\|u_{p_{n}}\right\|_{\infty}<\sqrt{e}
$$

Now, we will show that for large $p_{n}$, (3.13) implies the following estimate:

$$
z_{n}(x) \leq C+\log \frac{1}{\left(1+\frac{|x|^{2}}{8}\right)^{2}} \quad \forall x \in \Omega_{p_{n}}
$$

where $C$ is a constant independent of $n$.

By Theorem 1.1, $z_{n} \rightarrow z$ in $C^{\circ}\left(\bar{B}_{1}\right)$ and hence (3.14) fails for $x \in B_{1}$. It is enough to prove 3.14 for $x \in \Omega_{n} \backslash B_{1}$. To prove this let us observe that the function $z$ satisfies

$$
-\Delta z=e^{z} \geq\left(1+\frac{z}{p}\right)^{p}
$$

for any $p>1$. Furthermore, let us consider $\psi_{n}=z_{n}-z$ in $\Omega_{p_{n}}$. By computing $\psi_{n}$ on $\partial\left(\Omega_{p_{n}} \backslash B_{1}\right)$ and by applying the maximum principle, if $x \in \partial \Omega_{p_{n}}$, we get

$$
\begin{aligned}
\psi_{n}(x) & =z_{n}(x)-z(x)=-p_{n}+2 \log \left(1+\frac{|x|^{2}}{8}\right) \leq-p_{n}+2 \log \frac{1}{\varepsilon_{p_{n}}^{2}}+C \\
& \leq-p_{n}+2 \log u_{p_{n}}\left(x_{p_{n}}\right)^{p_{n}-1}+C \leq C
\end{aligned}
$$

where we had used $u_{p_{n}}\left(x_{p_{n}}\right)<\sqrt{e}$.

Now if $x \in \partial B_{1}$, by Theorem 1.1 we can derive again that $\psi_{n}(x) \leq C$.

Finally, we write down the equation satisfied by $\psi_{n}$. Using the convexity of $F(s)=\left(1+\frac{s}{p}\right)^{p}$ for $p>1$ we have

$$
\begin{aligned}
-\Delta \psi_{n} & =\left(1+\frac{z_{n}}{p_{n}}\right)^{p_{n}}-\left(1+\frac{z}{p_{n}}\right)^{p_{n}} \\
& \leq\left(1+\frac{z_{n}}{p_{n}}\right)^{p_{n}-1} \psi_{n}=\frac{u_{p_{n}}^{p_{n}-1}\left(\varepsilon_{p_{n}} x+x_{p_{n}}\right)}{u_{p_{n}}^{p_{n}-1}\left(x_{p_{n}}\right)} \psi_{n} .
\end{aligned}
$$

Since the maximum principle holds in $\Omega_{p_{n}} \backslash B_{1}$ for $L_{p_{n}, \Omega_{p_{n}} \backslash B_{1}}$, we now deduce that $\psi_{n} \leq C$ in $\Omega_{p_{n}} \backslash B_{1}$ and this gives (3.14).

From (3.14), a contradiction follows easily. Indeed, using Theorem 1.1 and Lebesgue's Theorem we derive

$$
\begin{aligned}
1 & =\int_{\Omega} u_{n}^{p_{n}+1}=u_{n}\left(x_{n}\right)^{p_{n}+1} \varepsilon_{n}^{2} \int_{\Omega_{n}}\left(1+\frac{z_{n}}{p_{n}}\right)^{p_{n}+1} \\
& =\frac{u_{n}^{2}\left(x_{n}\right)}{8 \pi e+o(1)}(8 \pi+o(1)),
\end{aligned}
$$

which proves that $\lim _{n \rightarrow \infty}\left\|u_{n}\right\|_{\infty}=\sqrt{e}$, a contradiction with (3.13).

\section{ACKNOWLEDGMENTS}

This work was done while the second author was visiting the TIFR centre in Bangalore. He would like to thank the School of Mathematics for its support and warm hospitality. 


\section{REFERENCES}

[1] Adimurthi and M. Struwe, Global compactness properties of semilinear elliptic equations with critical exponential growth, Journal of Functional Analysis 175 (2000), 125-167. MR 2001g:35063

[2] H. Brezis and F. Merle, Uniform estimates and blow-up behavior for solutions of $-\Delta u=$ $V(x) e^{u}$ in two dimensions, Comm. Partial Differential Equations 16 (1991), 1223-1253. MR 92m:35084

[3] W. Chen and C. Li, Classification of solutions of some nonlinear elliptic equations, Duke Math. J. 63 (1991), 615-622. MR 93e:35009

[4] G. Chen, W. M. Ni, and J. Zhou, Algorithms and visualization for solutions of nonlinear elliptic equations, Internat. J. Bifur. Chaos Appl. Sci. Engrg. 10 (2000), 1565-1612.

[5] K. El Medhi and M. Grossi, Asymptotic estimates and qualitative properties of an elliptic problem in dimension two, preprint.

[6] M. Flucher and J. Wei, Semilinear Dirichlet problem with nearly critical exponent, asymptotic location of hot spots, Manuscripta Math. 94 (1997), 337-346. MR 99b:35066

[7] D. Gilbarg and N. Trudinger, Elliptic partial differential equations of second order, Second edition, Grundlehren der mathematischen Wissenschaften, Band 224, Springer-Verlag, Berlin, 1983. MR 86c:35035

[8] X. Ren and J. Wei, On a two-dimensional elliptic problem with large exponent in nonlinearity, Trans. Amer. Math. Soc. 343 (1994), 749-763. MR 94h:35074

[9] X. Ren and J. Wei, Single-point condensation and least-energy solutions, Proc. Amer. Math. Soc. 124 (1996), 111-120. MR 96d:35037

[10] R. Schoen, On the number of constant scalar curvature metrics in a conformal class, "Differential geometry: A symposium in honor of Manfredo Do Carmo" (H. B. Lawson and K. Tenenblat, eds.), Longman Sci. Tech., Harlow, 1991, pp. 311-320. MR 94e:53035

T.I.F.R. Centre, P.O. Box 1234, Bangalore 560012, India

E-mail address: aditi@math.tifrbng.res.in

Università di Roma "La Sapienza", P.le Aldo Moro, 2, 00185 Roma, Italy

E-mail address: grossi@mat.uniroma1.it 